\title{
MOTIVACIÓN TEMPRANA HACIA EL APRENDIZAJE Y RENDIMIENTO LECTOR: ESTUDIO LONGITUDINAL
}

\author{
Rebeca Siegenthaler Hierro \\ Universitat Jaume I \\ siegenth@uji.es \\ Laura Abellán Roselló \\ Universitat Jaume I \\ Ana Badenes-Gasset Presentación \\ Universitat Jaume I \\ Jessica Mercader Ruiz \\ Universitat Jaume I
}

Fecha de Recepción: 5 Febrero 2019

Fecha de Admisión: 30 Abril 2019

\section{RESUMEN}

El objetivo del presente estudio longitudinal consistió en examinar el poder predictivo de distintas variables de motivación hacia el aprendizaje, evaluadas en Educación Infantil sobre diferentes aspectos del rendimiento en lectura en $2^{\circ}$ de Educación Primaria. La muestra inicial estuvo compuesta por 209 niños de Educación Infantil de 5 a 6 años y sus tutores, de los cuales 179 volvieron a ser evaluados dos años más tarde. En Educación Infantil, Ios maestros y maestras cumplimentaron la escala de estimación Preschool Learning Behaviors Scale (PLBS; McDermott, Green, Francis, y Stott, 2000) que evalúa las conductas de aprendizaje de competencia-motivación (conductas relacionadas con la anticipación del éxito), atención-persistencia (capacidad de persistir realizando una tarea hasta llegar a completarla) y actitud hacia el aprendizaje (voluntad de participar en las actividades de aprendizaje). Dos años más tarde, se administró la Batería de Evaluación de los Procesos Lectores (PROLEC-R; Cuetos, Rodríguez, Ruano, y Arribas, 2007) para evaluar diferentes aspectos relacionados con el rendimiento lector: procesos de identificación de letras, de acceso al léxico y de procesos sintácticos y semánticos. Los resultados mostraron que las variables de motivación hacia el aprendizaje evaluadas en Educación Infantil son capaces de predecir el rendimiento lector posterior, con un especial peso de la capacidad para persistir ante las tareas. Se comentan las implicaciones de estos hallazgos para la investigación y la práctica psicoeducativa.

Palabras clave: estudio Iongitudinal; rendimiento lector; motivación; competencia percibida; persistencia; actitud hacia el aprendizaje 


\begin{abstract}
Early motivation toward learning and performance reader: Iongitudinal study. The aim of the present longitudinal study was to examine the predictive power of different variables of motivation towards learning, evaluated in Kindergarten on different aspects of reading performance in the 2nd grade of Primary School. The initial sample consisted of 209 pre-schoolers from 5 to 6 years old and their teachers, of whom 179 were evaluated again two years later. In Kindergarten, teachers completed the Preschool Learning Behaviours Scale (PLBS, McDermott, Green, Francis, \& Stott, 2000 ) that assesses competency-motivation learning behaviours (behaviours related to the anticipation of success), attention-persistence (ability to persist doing a task until it is completed) and attitude towards learning (willingness to participate in learning activities). Two years later, a Spanish standardized assessment of reading performance (PROLEC-R, Cuetos et al., 2007) was administered to evaluate different aspects related to reading performance: letter identification, access to the lexicon, syntactic and semantic processes. The results showed that the variables of motivation towards learning evaluated in Kindergarten are able to predict the subsequent reading performance, with a special weight of the ability to persist doing a task until it is completed. The implications of these findings for research and psychoeducational practice are discussed.
\end{abstract}

Keywords: Iongitudinal study; reader performance; motivation; perceived competence; persistence; attitude towards learning

\title{
ANTECEDENTES DE LA TEMÁTICA A TRATAR
}

El rendimiento lector se considera "el proceso de extraer y construir significado simultáneamente a través de la interacción e implicación con el texto" (Snow, 2002, p.11) Requiere una serie de pasos, como procesar información para el reconocimiento de letras y palabras, encontrar la idea principal, entender las reglas, signos sintácticos y gramaticales 0 evaluar el contenido (Susanti, Buan, y Suhartono, 2013). Todo ello es un proceso consciente que implica intención de aprender. Por esto, no es correcto pensar que el rendimiento lector es una habilidad que depende sólo de factores cognitivos. En la lectura también influyen factores afectivo-motivacionales como la confianza en sí mismo o la actitud (Kusdemir y Bulut, 2018).

La motivación se asocia al modo en el que se inicia, se sostiene y se dirige una misma conducta, y a la reacción que provoca en el sujeto cuando realiza la actividad (Míguez, 2005). Dentro del plano educativo, Ajello (2003) se refiere a la motivación como la predisposición para aprender, cada vez de forma más autónoma. Las percepciones, actitudes y expectativas del alumno sobre sí mismo, sobre la tarea y sobre las metas que pretende alcanzar, como señalan García-Bacete y Doménech (1997), condicionan y guían su conducta en el ámbito escolar. La relación existente entre la motivación hacia el aprendizaje y el rendimiento lector ha sido investigada utilizando muestras de niños de Educación Primaria (McKenzie, Gow, y Schweitzer, 2004; Pinxten, Marsh, De Fraine, Van Der Noortgate, y Van Damme, 2014) y Secundaria (Moenikia y Zahed-Babelan, 2010; Suárez-Álvarez, Fernández, y Muñiz, 2014). Por ejemplo, Schiefele, Stutz y Schaffner (2016) con alumnado de Educación Primaria de primer y tercer curso, encontraron que la motivación para aprender, tras un intervalo de un año, predecía el rendimiento lector, concretamente de la comprensión lectora. En secundaria, los resultados obtenidos por Miñano y Castejón (2008) también confirman el poder predictivo de diferentes variables motivaciones sobre el rendimiento académico posterior en lenguaje.

Los trabajos son más escasos con muestras de niños menores de 7 años, como destacan Mokrova, O'Brien, Calkins, Leerkes y Marcovitch (2013), poniendo de manifiesto la necesidad de llevar a cabo más estudios longitudinales desde las primeras etapas referidos a la relación entre la motivación y las competencias académicas. Algunas investigaciones definen esta motivación de los 
niños más pequeños con el término "learning behaviors", refiriéndose a conductas de aprendizaje que son directamente observables (McDermott, Mordell, Stoltzfus, 2001). Destacan los trabajos realizados por el equipo de McDermott con niños de familias desfavorecidas. Estos estudios muestran que concretamente la autocompetencia percibida, la persistencia ante los errores y la actitud hacia el aprendizaje que aparecen en Educación Infantil, tienen un peso importante como predictores del rendimiento académico posterior en todas las áreas (Fantuzzo, Perry, y McDermott, 2004; McDermott et al., 2001). Es más, para estos investigadores la autocompetencia percibida y la persistencia en las tareas, son los dos grandes predictores del éxito escolar. Dichas conductas determinan el grado de esfuerzo y compromiso que los alumnos muestran para dirigirse a una meta en las tareas del aula (McDermott et al., 2000). Además, las conductas referidas a la anticipación del éxito en base a la competencia percibida, actúan como un factor de protección para dificultades de aprendizaje que puedan aparecer en un futuro (McDermott, Goldberg, Watkins, Stanley, y Glutting, 2006; McDermott et al., 2011).

En definitiva, son muchos los estudios sobre motivación en el contexto escolar, pero muy pocos los que han estudiado la influencia de esta variable en niños pequeños, entre los que predominan Ios trabajos realizados dentro del programa "Head Start Children" (Department of Health and Human Services, USA). Estos estudios se han centrado en niños pequeños, pero de familias con un nivel socio-económico bajo. Es por ello que faltan investigaciones de carácter longitudinal, utilizando muestras de población general, que profundicen en la motivación hacia el aprendizaje en niños pequeños y su valor predictivo sobre el rendimiento lector posterior. Estudios longitudinales que identifiquen el peso de variables motivacionales desde la etapa de Educación Infantil en la predicción del rendimiento lector futuro.

\section{OBJETIVOS DE LA INVESTIGACIÓN}

El objetivo del presente estudio longitudinal fue examinar el poder predictivo de diferentes variables de motivación inicial hacia el aprendizaje (motivación-competencia, atención-persistencia y actitud hacia el aprendizaje) evaluadas en Educación Infantil (T1) sobre distintos procesos implicados en el rendimiento en lectura en $2^{\circ}$ de Educación Primaria (T2).

\section{MUESTRA Y/O PARTICIPANTES}

La muestra del estudio se seleccionó siguiendo un procedimiento de muestreo aleatorio simple. No formaron parte del estudio sujetos con un $\mathrm{Cl}$ equivalente inferior a 70 , ni aquéllos cuyos informes escolares reflejaran la presencia de deprivación socio-cultural, deficiencias sensoriales, anomalías neurobiológicas y/o problemas psicológicos graves. La muestra definitiva del estudio estuvo conformada por 179 estudiantes ( $51.1 \%$ varones; $49.9 \%$ niñas) y sus maestros/as, pertenecientes a 13 centros educativos ( $63.5 \%$ centros públicos; $36.5 \%$ centros concertados).

En el T1 $(n=208)$, los participantes tenían edades comprendidas entre 5 y 6 años [Media (meses) $=70.21$, DT $=3.56$ ]. Se obtuvo el $\mathrm{Cl}$ equivalente $[$ Media $=98.28$; DT $=12.34$; rango $=70$ 128] a partir de las subpruebas de Vocabulario y Cuadrados del WPPSI (Wechsler, 1996), siguiendo las directrices de Spreen y Strauss (1991). El 88\% de los sujetos era de nacionalidad española. Todos los participantes hablaban y comprendían el castellano.

En el T2 ( $n=179,86.6 \%$ de la muestra inicial), los sujetos tenían edades comprendidas entre 7 y 8 años [Media (meses) = 94.16; DT = 3.48]. El 19.1\% de los participantes asistían a sesiones con especialistas en los respectivos centros escolares: Apoyo Educativo (7.7\%), Educación Compensatoria (1.9\%), Pedagogía Terapéutica $(3.3 \%)$, Audición y Lenguaje $(3.8 \%)$ y tratamiento combinado $(2.4 \%)$. 


\section{MOTIVACIÓN TEMPRANA HACIA EL APRENDIZAJE Y RENDIMIENTO LECTOR: ESTUDIO LONGITUDINAL}

\section{METODOLOGÍA}

\section{Instrumentos}

T1: Motivación inicial hacia el aprendizaje

Para evaluar las conductas de motivación hacia el aprendizaje en Educación Infantil los tutores/as cumplimentaron la escala de estimación Preeschool Learning Behaviour Scale (PLBS; McDermott, Green, Francis, y Stott, 2000). El cuestionario se compone de 29 ítems con una escala Likert de respuesta ( 0 = "Muy a menudo", 1 = "A veces", 2 = "Casi nunca"), que se agrupan en 3 subescalas: competencia-motivación, que evalúa las conductas relacionadas con la anticipación del éxito (i.e. "Parece que se refugia en una actitud de impotencia"); atención-persistencia, centrada en la capacidad de persistir realizando una tarea hasta llegar a completarla; (i.e. "Se implica en las tareas en la medida que se espera que lo hiciera para su edad"); y actitud hacia el aprendizaje, cuyos ítems se refieren a la voluntad de participar en las actividades de aprendizaje, mostrando una actitud positiva hacia los elementos que lo conforman (i.e. "Muestra poco interés en agradar al profesor"). Se constató el nivel de fiabilidad de la escala para la presente muestra (Alfa de Cronbach = .86). Se utilizó la puntuación directa en cada una de las subescalas para los análisis.

\section{T2: Rendimiento lector}

Para evaluar el rendimiento lector en $2^{-}$curso de Educación Primaria, se administró la Batería de Evaluación de los Procesos Lectores, Revisada (PROLEC-R; Cuetos et al., 2007). La prueba está compuesta por 8 tareas que evalúan cuatro procesos lectores de distinta complejidad: identificación de letras (identificación de letras e igual-diferente o discriminación fonológica); procesos léxicos (lectura de palabras y pseudopalabras); procesos sintácticos (identificación de distintas estructuras gramaticales y lectura de un texto con signos de puntuación); y procesos semánticos (comprensión de oraciones y comprensión de textos). La batería ofrece tanto puntuaciones globales (índices principales), como puntuaciones de aciertos (índices de precisión) y tiempo de lectura (índices de velocidad) para determinadas tareas (nombre de las letras, igual-diferente, lectura de palabras, lectura de pseudopalabras y signos de puntuación). La fiabilidad test-retest de la prueba se ha constatado en .79, además de contar con elevados índices de validez (Cuetos et al., 2007). Para los análisis, se utilizaron las puntuaciones directas obtenidas en los índices principales, de precisión y velocidad para cada una de las tareas.

\section{Procedimiento}

La presente investigación fue autorizada por la Consellería de Educación, Cultura y Deporte y el Comité Ético de la Universitat Jaume I. Igualmente, se contó con el permiso de los centros educativos y el consentimiento informado de padres/madres/tutores previa participación en el estudio. En Educación Infantil, los maestros/as cumplimentaron el cuestionario PLBS (McDermott et al., 2000). Dos años después, se retornó a los centros escolares para la administración de la batería PROLEC$R$ (Cuetos et al., 2007) a los estudiantes. La evaluación fue llevada a cabo por profesionales experimentados en aulas habilitadas por los centros escolares que reunían condiciones óptimas de iluminación, sonoridad y ventilación. La administración de las pruebas comprendió dos sesiones individuales de 25 minutos en horario escolar que no interferían con las actividades significativas del curriculum.

\section{Análisis estadísticos}

Se utilizó el paquete estadístico Statistical Package for the Social Science, versión 22.00 (SPSS Inc., 2013), para realizar los análisis estadísticos. Se realizaron análisis de regresión lineal múltiple por el método de pasos sucesivos con el fin de comprobar qué conductas de motivación hacia el 
aprendizaje evaluadas en el T1 predecían las distintas subpruebas que conforman los procesos implicados en el rendimiento lector en el T2.

\section{RESULTADOS ALCANZADOS}

En la Tabla 1 se recogen los análisis de regresión de las distingas variables de motivación hacia el aprendizaje sobre los índices principales de la batería PROLEC-R (Cuetos et al., 2007). La subescala de atención-persistencia resultó ser el único predictor estadísticamente significativo para los procesos léxicos (lectura de palabras, $\Delta R^{2}=.168, p<.001$; lectura de pseudopalabras $\Delta R^{2}=.110$, $p<.001)$, así como para las tareas de nombre de las letras $\left(\Delta R^{2}=.152, p<.001\right)$, signos de puntuación $\left(\Delta R^{2}=.290, p<.001\right)$ y comprensión de textos $\left(\Delta R^{2}=.085, p<.001\right)$. La variable competencia-motivación fue el único predictor estadísticamente significativo para las tareas de igualdiferente $\left(\Delta R^{2}=.150, p<.001\right)$ y estructuras gramaticales $\left(\Delta R^{2}=.133, p<.001\right)$. El $23.3 \%$ de la varianza de la tarea de comprensión de textos fue predicho por ambas variables (atención-persistencia, $\Delta R^{2}=.211, p<.001$; competencia-motivación, $\Delta R^{2}=.022, p=.024$ ). La subescala actitud hacia el aprendizaje no alcanzó la significación estadística en la predicción de los índices principales en ninguno de los casos.

Tabla 1. Resultados del análisis de regresión de las variables de motivación hacia el aprendizaje evaluadas en el T1 sobre los índices principales de las tareas de rendimiento lector en el T2

\begin{tabular}{|c|c|c|c|c|c|}
\hline \multirow{3}{*}{ Procesos } & \multirow[b]{3}{*}{ Tareas/Predictores } & \multicolumn{4}{|c|}{ ÍNDICES PRINCIPALES (TOTAL) } \\
\hline & & $\mathrm{F}$ & $R^{2}$ & $\Delta R^{2}$ & Beta \\
\hline & & & & & \\
\hline \multirow{3}{*}{$\begin{array}{l}\text { Identificación } \\
\text { de letras }\end{array}$} & Nombre de las letras & & & & \\
\hline & Atención-Persistencia & $31.77 * *$ & .152 & .152 & .390 \\
\hline & $\begin{array}{l}\text { Competencia-Motivación } \\
\text { Lectura de palabras }\end{array}$ & $31.10 * *$ & .150 & .150 & .388 \\
\hline \multirow[t]{2}{*}{ Procesos léxicos } & $\begin{array}{c}\text { Atención-Persistencia } \\
\text { Lectura de pseudopalabras }\end{array}$ & $35.78 * *$ & .168 & .168 & .410 \\
\hline & $\begin{array}{l}\text { Atención-Persistencia } \\
\text { Estructuras gramaticales }\end{array}$ & $21.93 * *$ & .110 & .110 & .332 \\
\hline \multirow[t]{2}{*}{$\begin{array}{l}\text { Procesos } \\
\text { sintácticos }\end{array}$} & $\begin{array}{l}\text { Competencia-Motivación } \\
\text { Signos de puntuación }\end{array}$ & $27.26^{* *}$ & .133 & .133 & .365 \\
\hline & $\begin{array}{l}\text { Atención-Persistencia } \\
\text { Comprensión de oraciones }\end{array}$ & $72.13 * *$ & .290 & .290 & .538 \\
\hline \multirow[t]{2}{*}{$\begin{array}{l}\text { Procesos } \\
\text { semánticos }\end{array}$} & $\begin{array}{l}\text { Atención-Persistencia } \\
\text { Competencia-Motivación } \\
\text { Comprensión de textos }\end{array}$ & $26.79 * *$ & 233 & $\begin{array}{l}.211 \\
.022\end{array}$ & $\begin{array}{l}.262 \\
.248\end{array}$ \\
\hline & Atención-Persistencia & $16.44 * *$ & .085 & .085 & .292 \\
\hline
\end{tabular}

Nota: $*=p<.05 ; * * p<.001$

En lo que se refiere a las regresiones sobre los índices de precisión (véase Tabla 2), la subescala atención-persistencia resultó ser el único predictor estadísticamente significativo para las tareas relativas a los procesos léxicos (lectura de palabras, $\Delta R^{2}=.162, p<.001$; lectura de pseudopalabras, $\left.\Delta R^{2}=.114, p<.001\right)$. La subescala competencia-motivación, fue el único predictor estadísticamente significativo para el índice de precisión de la tarea de nombre de las letras $\left(\Delta R^{2}=.226\right.$, $p<.001)$. Ambas subescalas, predijeron el $21.4 \%$ de la varianza de los índices de precisión de las 


\section{MOTIVACIÓN TEMPRANA HACIA EL APRENDIZAJE Y RENDIMIENTO LECTOR: ESTUDIO LONGITUDINAL}

tareas de igual-diferente (competencia-motivación, $\Delta R^{2}=.195, p<.001$; atención-persistencia, $\Delta R^{2}=.019, p=.040$ ), así como el $32.1 \%$ de la tarea de signos de puntuación (atención-persistencia, $\Delta R^{2}=.300, p<.001$; competencia-motivación, $\Delta R^{2}=.021, p=.022$ ). La subescala de actitud hacia el aprendizaje no resultó ser un predictor estadísticamente significativo para ninguno de los índices de precisión.

Tabla 2. Resultados del análisis de regresión de las variables de motivación hacia el aprendizaje evaluadas en el T1 sobre los índices de presión de las tareas de rendimiento lector en el T2

\begin{tabular}{|c|c|c|c|c|}
\hline & \multicolumn{4}{|c|}{ ÍNDICES DE PRECISIÓN (ACIERTOS) } \\
\hline & $\mathrm{F}$ & $R^{2}$ & $\Delta R^{2}$ & Beta \\
\hline \multicolumn{5}{|l|}{ Tareas/Predictores } \\
\hline \multicolumn{5}{|l|}{ Nombre de las letras } \\
\hline Competencia-Motivación & $51.63^{* *}$ & 226 & .226 & .475 \\
\hline \multicolumn{5}{|l|}{ Igual-Diferente } \\
\hline Competencia-Motivación & & & .195 & .260 \\
\hline Atención-Persistencia & $23.83^{* *}$ & .214 & .019 & .228 \\
\hline \multicolumn{5}{|l|}{ Lectura de palabras } \\
\hline Atención-Persistencia & $34.14^{* *}$ & .162 & .162 & .402 \\
\hline \multicolumn{5}{|l|}{ Lectura de pseudopalabras } \\
\hline Atención-Persistencia & $22.72^{* *}$ & .114 & .114 & .337 \\
\hline \multicolumn{5}{|l|}{ Signos de puntuación } \\
\hline Atención-Persistencia & & & .300 & .360 \\
\hline Competencia-Motivación & $41.49^{* *}$ & .321 & .021 & .237 \\
\hline
\end{tabular}

Respecto a las regresiones sobre los índices de velocidad (véase Tabla 3), la subescala de atención-persistencia fue el único predictor estadísticamente significativo para la tarea de nombre de las letras $\left(\Delta R^{2}=.114, p<.001\right)$. La subescala de competencia-motivación, predijo significativamente los índices de velocidad de las tareas igual-diferente $\left(\Delta R^{2}=.151, p<.001\right)$ y signos de puntuación $\left(\Delta R^{2}=.140, p<.001\right)$. La subescala de actitud hacia el aprendizaje fue el único predictor significativo para el índice de velocidad de la tarea de lectura de pseudopalabras $\left(\Delta R^{2}=.065, p=.001\right)$. El $23.6 \%$ de la varianza del índice de velocidad de la tarea de lectura de palabras fue predicho por ambas subescalas (competencia-motivación, $\Delta R^{2}=.216, p<.001$; atención-persistencia $\Delta R^{2}=$ $.021, p=.030)$. 
Tabla 3. Resultados del análisis de regresión de las variables de motivación hacia el aprendizaje evaluadas en el T1 sobre los índices de velocidad de las tareas de rendimiento lector en el T2

\begin{tabular}{lcccc}
\hline & \multicolumn{4}{c}{ ÍNDICES DE VELOCIDAD (TIEMPO) } \\
\cline { 2 - 5 } & $\mathrm{F}$ & $R^{2}$ & $\Delta R^{2}$ & Beta \\
\cline { 2 - 5 } & & & & \\
$\begin{array}{c}\text { Nombre de las letras } \\
\quad \text { Atención-Persistencia }\end{array}$ & $22.68^{* *}$ & .114 & .114 & -.337 \\
$\begin{array}{c}\text { Igual-Diferente } \\
\quad \text { Competencia-Motivación }\end{array}$ & $31.34^{* *}$ & .151 & .151 & -.389 \\
$\begin{array}{c}\text { Lectura de palabras } \\
\quad \text { Competencia-Motivación }\end{array}$ & & & .216 & -.275 \\
$\quad \begin{array}{c}\text { Atención-Persistencia } \\
\text { Lectura de pseudopalabras } \\
\quad \text { Actitud hacia el aprendizaje }\end{array}$ & $27.26^{* *}$ & .236 & .021 & -.238 \\
$\begin{array}{c}\text { Signos de puntuación } \\
\text { Competencia-Motivación }\end{array}$ & $12.30^{*}$ & .065 & .065 & -.255 \\
\hline
\end{tabular}

Nota: $*=p<.05 ; * * p<.001$

\section{DISCUSIÓN}

Este estudio analiza el poder predictivo que tienen diferentes variables motivacionales hacia el aprendizaje evaluadas a los 5 años, mediante el cuestionario PLBS (McDermott et al., 2000) cumplimentado por los maestros/as, sobre el rendimiento lector dos cursos académicos después. Los resultados muestran que las variables motivacionales tienen un peso significativo en la predicción de las puntuaciones globales (índices principales) de todas las habilidades lectoras evaluadas, con varianzas que oscilan entre el $8.5 \%$ y el $29 \%$. Estos resultados respaldan la idea de que desde edades tempranas, variables motivaciones como la actitud, el esfuerzo y la perseverancia, y una autocompetencia adecuada, son indicadores del rendimiento lector posterior, como han destacado autores como Ladd, Buhs y Seid (2000), McDermott et al. (2011) y McWayne, Green y Fantuzzo (2009).

Entre todas las variables analizadas destaca el poder predictivo de la persistencia, que ha demostrado ser un predictor estadísticamente significativo para las habilidades y tareas lectoras relacionadas con identificar el nombre de las letras, leer palabras y pseudopalabras, leer signos de puntuación y comprender oraciones y textos, resultado este último coincidente con Schiefele et al. (2016). Los valores de predicción de la persistencia más elevados son los obtenidos sobre las pruebas de signos de puntuación, con un $29 \%$ de la varianza explicada, y comprensión de oraciones, con un $21.1 \%$. Este resultado sobre el importante papel de la persistencia como predictor de rendimiento lector es coincidente con un estudio de carácter longitudinal con niños más pequeños (Mokrova et al., 2013). Los niños más persistentes a la hora de realizar una tarea con 3 años, desarrollaban mejores habilidades académicas en lectura dos años más tarde. Los investigadores concluyen que la persistencia del alumnado en la etapa de Educación Infantil es muy importante, independientemente de sus características socio-demográficas y habilidades cognitivo-lingüísticas.

La autocompetencia percibida también ha mostrado un peso notable como predictor de los aciertos (índice de precisión) en diversas tareas lectoras. Concretamente, la autocompetencia percibida predice un porcentaje significativo de la varianza de la subprueba de signos de puntuación (30\%), así como de las dos tareas de reconocimiento de letras (nombre de letras, 22.6\%; igual-diferente, $19.5 \%$ ). Esta variable motivacional ha sido igualmente el mayor predictor de la velocidad lectora (índice de velocidad) en la tarea de lectura de palabras, explicando hasta un $21.6 \%$ de la varian- 


\section{MOTIVACIÓN TEMPRANA HACIA EL APRENDIZAJE Y RENDIMIENTO LECTOR: ESTUDIO LONGITUDINAL}

za. Estos resultados están en la línea de García-Bacete y Doménech (1997) en el sentido de que las percepciones, actitudes y expectativas del alumno y alumna sobre sí mismo son un elemento fundamental que guía y condiciona su conducta en el contexto escolar. Mucho menores son los resultados de la variable actitud, que muestra únicamente un poder limitado de predicción sobre la velocidad en la subprueba de lectura de pseudopalabras (6.5\%). La escasez de resultados en esta variable podría deberse a la escasa experiencia escolar de los niños y niñas. Es probable que a esta temprana edad la actitud hacia el aprendizaje no sea todavía consistente y esté todavía muy ligada a los acontecimientos del día a día.

En síntesis, se concluye que la autocompetencia y la persistencia tienen un peso significativo en la predicción del rendimiento posterior. Este resultado es coincidente con los obtenidos por el equipo de McDermott con niños de familias desfavorecidas (Fantuzzo, et al., 2004; McDermott et al., 2000; McDermott et al., 2001; McDermott et al., 2006; McDermott et al., 2011). El presente trabajo resulta innovador, puesto que es un estudio longitudinal y presenta una muestra conformada por niños de Educación Infantil de la población general. Como limitación hay que destacar que la herramienta utilizada para evaluar la motivación del alumnado es un cuestionario que se administró a los maestros. Sería interesante incorporar otros métodos de evaluación en los que la motivación se evalúe a través del propio niño o niña, o por otras personas cercanas. Futuras investigaciones, deberían tener en cuenta también una serie de variables que no se han contemplado aquí, tanto contextuales y socioambientales (la familia como agente educativo y el contexto donde se desarrolla el niño) como instruccionales e institucionales (influencia del docente sobre la motivación y expectativas del alumno, relaciones con sus iguales, etc.). Asimismo, tampoco se han considerado variables personales, como la implicación en el rendimiento de los aspectos cognitivos y del funcionamiento ejecutivo.

Es importante que tanto las familias como el personal docente conozcan la importancia que tiene la motivación en el futuro rendimiento de sus hijos o alumnos. Resulta relevante que, tanto en los hogares como en las aulas, se propicien ambientes de aprendizaje que fomenten el protagonismo de los niños. Además, aspectos como crear ambientes estables, seguros y agradables también favorecerán el gusto por el aprendizaje en sí. Los materiales y las actividades son también aspectos importantes a tener en cuenta (Ryan y Deci, 2000; Shernoff y Csikszentmihaly, 2009). Proporcionar textos interesantes y retroalimentación positiva con respecto a la comprensión es también fundamental para fortalecer la actitud del niño sobre la relevancia y el valor de la lectura (Guthrie y Klauda, 2014; Guthrie y Wilgfield, 2005).

\section{CONCLUSIONES}

Se concluye que la motivación hacia el aprendizaje en Educación Infantil tiene una influencia notable sobre el rendimiento lector posterior, especialmente la autocompetencia y la persistencia. La autocompetencia percibida tiene un papel especialmente relevante en la predicción de los procesos de reconocimiento de letras, mientras que la persistencia es la variable con más peso en los procesos léxicos, sintácticos y semánticos.

\section{REFERENCIAS BIBLIOGRÁFICAS}

Ajello, A. M. (2003). La motivación para aprender. En C. Pontecorvo (Coord.), Manual de psicología de la educación (pp. 251-271). Madrid: Popular.

Cuetos, F., Rodríguez, B., Ruano, E. y Arribas, D. (2007). PROLEC-R, Batería de Evaluación de los Procesos Lectores, Revisada. (4⿳亠丷厂二 Edición). Madrid: TEA Ediciones.

Fantuzzo, J., Perry, M. A., y McDermott, P. (2004). Preschool Approaches to Learning and Their Relationship to Other Relevant Classroom Competencies for Low-Income Children. School 
Psychology Quarterly, 19(3), 212.

García-Bacete, F. J., y Doménech, F. (1997). Motivación, aprendizaje y rendimiento escolar. Revista Electrónica de Motivación y Emoción (R.E.M.E), 1(0).

Guthrie, J. T., y S. L. Klauda. (2014). Effects of Classroom Practices on Reading Comprehension, Engagement, and Motivations for Adolescents. Reading Research Quarterly, 49(4), 87-416.

Guthrie, J.T., y Wigfield A. (2005). Roles of motivation and engagement in reading comprehension assessment. En S. G. Paris y S.A. Stahl, (Eds), Children's Reading Comprehension and Assessment (pp.187-213). Mahway, NJ: Lawrence Erlbaum Associates

IBM Corp. Released (2013). IBM SPSS Statistics for Windows, Version 22,0. Armonk, NY: IBM Corp

Kusdemir, Y., y Bulut, P. (2018). The Relationship between Elementary School Students' Reading Comprehension and Reading Motivation. Journal of Education and Training Studies, 6(12), 97110

Ladd, G., Buhs, E., y Seid, M. (2000). Children's initial sentiments about kindergarten: Is school liking an antecedent of early childhood participation and achievement? Merrill-Palmer Quarterly, 46, 255-279

McDermott, P. A., Goldberg, M. M., Watkins, M. W., Stanley, J. L., y Glutting, J. J. (2006). A nationwide epidemiologic modeling study of LD: Risk, protection, and unintended impact. Journal of Learning Disabilities, 39(3), 230-251.

McDermott, P. A., Green, L. F., Francis, J. M., y Stott, D. H. (2000). PLBS; Preschool Learning Behaviors Scale. Philadelphia: Edumetric \& Clinical Science.

McDermott, P. A., Mordell, M., y Stoltzfus, J. C. (2001). The organization of student performance in American schools: Discipline, motivation, verbal learning, nonverbal learning. Journal of Educational Psychology, 93(1), 65-76.

McDermott, P. A., Fantuzzo, J. W., Warley, H. P., Waterman, C., Angelo, L. E., Sekino, S., y Gadsden, V. L. (2011). Multidimensionality of teachers' gradedd responses for preschoolers' stylistic learning behavior: The Learning-To-Learn Scales. Educational and Psychological Measurement, 71, 148-169.

McKenzie, K., Gow, K., y Schweitzer, R. (2004). Exploring first year academic achievement through structural equation modelling. Higher Education Research \& Development, 23(1), 95-112.

McWayne, C., Green, L., y Fantuzzo, J. (2009). A variable- and person-oriented investigation of preschool competencies and Head Start children's transition to kindergarten and first grade. Applied Developmental Science, 13, 1-15.

Míguez, M. (2005). Motivación y comprensión-motivación y aprendizaje. Revista ieRed: Revista electrónica de la red de investigación educativa, 1(3).

Miñano, P., y Castejón, J. L. (2008). Capacidad predictiva de las variables cognitivo-motivacionales sobre el rendimiento académico. REME. Revista Electrónica de Motivación y Emoción, 28(11).

Moenikia, M., y Zahed-Babelan, A. (2010). A study of simple and multiple relations between mathematics attitude, academic motivation and intelligence quotient with mathematics achievement. Procedia-Social and Behavioral Sciences, 2(2), 1537-1542.

Mokrova, I. L., O'Brien, M., Calkins, S. D., Leerkes, E. M., y Marcovitch, S. (2013). The role of persistence at preschool age in academic skills at kindergarten. European journal of psychology of education, 28(4), 1495-1503.

Pinxten, M., Marsh, H. W., De Fraine, B., Van Den Noortgate, W., y Van Damme, J. (2014). Enjoying mathematics or feeling competent in mathematics? Reciprocal effects on mathematics achievement and perceived math effort expenditure. British Journal of Educational Psychology, 84(1), 152-174. 


\section{MOTIVACIÓN TEMPRANA HACIA EL APRENDIZAJE Y RENDIMIENTO LECTOR: ESTUDIO LONGITUDINAL}

Ryan, R., y Deci, E. (2000). Self-determination theory and the facilitation of intrinsic motivation, social development, and well-being. American Psychologist, 55, 68-78.

Schiefele, U., Stutz, F., y Schaffner, E. (2016). Longitudinal relations between reading motivation and reading comprehension in the early elementary grades. Learning and Individual Differences, 51, 49-58.

Shernoff, D., y Csikszentmihalyi, M. (2009). Flow in schools: Cultivating engaged learners and optimal learning environments. En R. Gilman, E. S. Huebner, y M. Furlong (Eds.), Handbook of Positive Psychology in Schools (pp. 131-146). New York, NY: Routledge

Snow, C. (2002). Reading for Understanding. Toward a Research and Development Program in Reading Comprehension. Santa Monica, CA: RAND.

Spreen, 0., y Strauss, E. (1991). A compendium of neuropsychological tests: Administration, norms and commentary. New York: Oxford University Press.

Suárez-Álvarez, J., Fernández-Alonso, R., y Muñiz, J. (2014). Self-concept, motivation, expectations, and socioeconomic level as predictors of academic performance in mathematics. Learning and Individual Differences, 30, 118-123.

Susanti, I., Buan, S., y Suhartono, L. (2013). The use of cloze procedure to test the students reading comprehension. Jurnal Pendidikan dan Pembelajaran, 2(2).

Wechsler, D. (1996). WPPSI; Escala de Inteligencia de Wechsler para Preescolar y Primaria. Madrid: TEA Ediciones. 